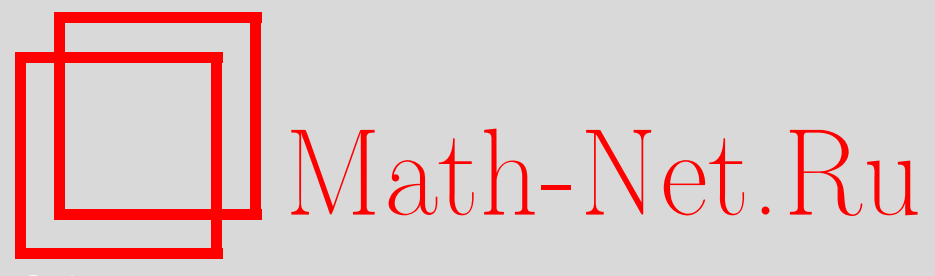

А. Е. Деревянка, Быстрая оценка минимального расстояния между двумя конфокальными гелиоцентрическими орбитами, Вестн. Сам. гос. техн. ун-та. Сер. Физ.-мат. науки, 2014, выпуск 4(), 144-156

DOI: https://doi.org/10.14498/vsgtu1344

Использование Общероссийского математического портала MathNet.Ru подразумевает, что вы прочитали и согласны с пользовательским соглашением

http://www.mathnet.ru/rus/agreement

Параметры загрузки:

IP : 54.162 .127 .20

26 апреля 2023 г., 06:32:49

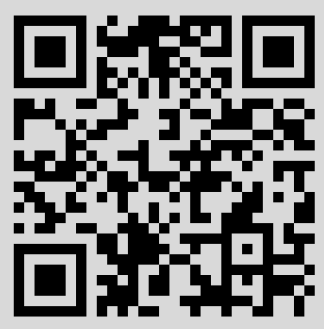




\title{
БЫСТРАЯ ОЦЕНКА МИНИМАЛЬНОГО РАССТОЯНИЯ МЕЖДУ ДВУМЯ КОНФОКАЛЬНЫМИ ГЕЛИОЦЕНТРИЧЕСКИМИ ОРБИТАМИ
}

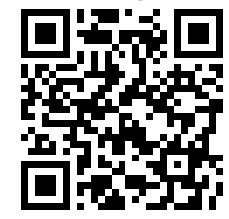

\section{A. Е. Деревянка}

Самарский государственный технический университет,

Россия, 443100, Самара, ул. Молодогвардейская, 244.

\begin{abstract}
Аннотация
Рассматривается один из аспектов задачи отнесения астероида к классу потенциально опасных для Земли астероидов, а именно, проблема оценки параметра MOID (Minimum Orbital Intersection Distance), характеризующего минимальное расстояние между двумя конфокальными гелиоцентрическими орбитами небесных тел. Рассмотрены аналитические, численные и численно-аналитические методы, применяемые для оценки параметра MOID. Дано краткое описание аналитических методов K. В. Холшевникова и G. F. Gronchi, считающихся классическими. Поставлена задача вычисления параметра MOID для большого количества астероидов (более 10000 ) с максимальной скоростью расчетов и возможностью параллелизации процесса. Предложен численный метод оценки, имеющий в основе геометрические соображения относительно расположения тел на орбитах. Рассматриваются два тела: $A$ и $E$. Так как в постановке задачи требуется рассчитать минимальное расстояние между орбитами, информация о фактических положениях тел на их орбитах не рассматривается. Для тела $A$ просчитывается полный оборот по орбите. Для каждого положения тела $A$ находится соответствующее ему положение тела $E$. Положение тела $E$ рассчитывается из следующего предположения. В рассмотрение вводится плоскость $P$, содержащая тело $A$, Солнце и перпендикулярная плоскости орбиты тела $E$. Из двух точек, в которых плоскость $P$ пересекает орбиту тела $E$, считается, что тело $E$ находится в ближайшей к телу $A$. Таким образом, положение тела $E$ будет зависеть от положения тела $A$. На основе геометрических соотношений из треугольника, образованного Солнцем и двумя телами, находится расстояние между телами $A$ и $E$. После просчета с определенным шагом одного полного оборота тела $A$ по орбите получается набор значений расстояний, из которого определяются области локальных минимумов дискретного представления функции расстояния между орбитами тел $A$ и $E$. Затем производится процедура уточнения найденных значений локальных минимумов дискретного представления функции расстояния. В итоге за минимальное расстояние между орбитами (параметр MOID) принимается наименьший из найденных локальных минимумов. Достоинства метода: высокая скорость и настраиваемая точ-
\end{abstract}

(C) 2014 Самарский государственный технический университет.

\section{Образец для цитирования}

Деревянка А. Е. Быстрая оценка минимального расстояния между двумя конфокальными гелиоцентрическими орбитами // Вестн. Сам. гос. техн. ун-та. Сер. Физ.-мат. науки, 2014. № 4 (37). С. 144-156. doi: 10.14498/vsgtu1344.

\section{Сведения об авторе}

Андрей Евгенъевич Деревлнка (AndrDerev@gmail.com), аспирант, каф. прикладной математики и информатики. 
ность вычислений, возможность использования параллельных вычислений. Проведены сравнительные испытания описываемого метода. Полученные результаты согласуются с классическим методом.

Ключевые слова: MOID, небесная механика, элементы орбит, потенциально опасные астероиды.

doi: http://dx.doi.org/10.14498/vsgtu1344

Проблема астероидной опасности включает в себя множество различных задач, одной из которых является отнесение астероида к классу потенциально опасных $[1,2]$. Согласно определению, используемому Лабораторией реактивного движения NASA (http://neo.jpl.nasa.gov/neo/groups.html), потенциально опасным астероидом (PHA - Potentially Hazardous Asteroid) считается такой, для которого одновременно выполняются два условия: орбита астероида имеет сближение с орбитой Земли на расстояние менее 0.05 a.e. (астрономических единиц) и диаметр астероида больше 150 метров (т.е. абсолютная звездная величина $H \leqslant 22)$. Для оценки расстояния между пересекающимися орбитами рассчитывается величина минимального расстояния между орбитами, сокращенно MOID (Minimum Orbital Intersection Distance).

В настоящее время астероидов, принадлежащих к группам Аполлона, Амура и Атона, насчитывается более 12 000. В основном потенциально опасные астероиды принадлежат именно к этим группам [3-5]. По данным, представленным Лабораторией реактивного движения NASA (http://neo.jpl. nasa.gov/orbits) и Центром малых планет (http://www . minorplanetcenter. net/iau/Dangerous.html), на сегодняшний день насчитывается более 1500 потенциально опасных астероидов.

Таким образом, при получении новых данных об элементах орбит астероидов необходимо произвести расчеты для выявления потенциально опасных. В силу того, что расчеты необходимо проводить для значительного числа астероидов, время расчетов становится важным критерием при выборе метода оценки MOID. Кроме того, важна и точность получаемых оценок.

Методы для оценки параметра MOID подразделяются на три группы: аналитические, численные и численно-аналитические.

Среди аналитических методов классическими считаются методы, созданные K. В. Холшевниковым [6,7] и G. F. Gronchi [8-10]. В работе К. В. Холшевникова [6] задача отыскания MOID сведена к решению тригонометрического уравнения восьмой степени, а также показано, что в общем случае дальнейшее упрощение задачи невозможно. В основе алгоритма, предложенного G. F. Gronchi, лежит быстрое преобразование Фурье. Задача сводится к алгебраическому полиному 16-й степени, действительные корни которого затем используются для отыскания критических точек функции, описывающей расстояние между орбитами $[9,10]$. Как метод К. В. Холшевникова, так и метод G. F. Gronchi в результате реализации предоставляют информацию не только о точках минимума функции расстояния между двумя орбитами, но и о других критических точках этой функции.

Методы, принадлежащие к группе численных, представляют собой итерационные алгоритмы. Задача нахождения MOID в них решается последовательным уточнением значения MOID. Достоинством численных методов является простота реализации и возможность настройки желаемой точно- 
сти и скорости работы. Схема работы методов этой группы состоит в следующем: сначала производится расчет расстояний между двумя точками на орбитах исследуемых тел, а потом проводится анализ дискретного представления функции расстояния между орбитами.

Большинство современных методов являются гибридными, удачно сочетая в себе достоинства численных и аналитических методов [9,11-15]. Численно-аналитические методы предоставляют высокую скорость с сохранением точности расчетов. Их общую структуру можно описать следующим образом. Аналитическими методами задача сводится к упрощенному виду, чтобы в дальнейшем применить численные методы для получения результата. В работах $[1,12,15-20]$ используется именно такой подход.

В данной работе представлен метод, позволяющий производить быструю оценку параметра MOID для двух тел на конфокальных эллиптических орбитах. Метод относится к группе численно-аналитических методов и предназначен для использования в случаях, когда требуется провести расчет параметра MOID для значительного числа астероидов, затратив при этом минимум времени. K примеру, такие расчеты необходимы в задаче оценки потенциальной опасности небесного объекта при оценке вероятности столкновения астероидов с Землей.

Оценка параметра MOID на основе данных наблюдений астероидов проводится для первичного отбора потенциально опасных астероидов и выделения астероидов, чьи орбиты расположены близко к орбите Земли. Для отобранных астероидов проводится более тщательное интегрирование уравнений движения. Кроме того, выделение неопасных астероидов необходимо по той причине, что процедура оценки вероятности столкновения астероида с Землей требует значительных вычислительных мощностей и проводить ее для всех астероидов групп Аполлона, Амура и Атона нецелесообразно. После первичного отбора потенциально опасных астероидов для оставшихся астероидов также возможно проведение оценки параметра MOID с начальными данными, вычисленными в результате интегрирования уравнений движения астероидов. Такая процедура дополнительной оценки может применяться для того, чтобы найти астероиды, переходящие в класс потенциально опасных в результате эволюции орбиты.

Единственным обязательным требованием метода, предъявляемым к исследуемым телам, является требование конфокальности эллиптических орбит. Введем обозначения для рассматриваемых небесных тел. Пусть имеются небесные тела $E$ и $A$. Положения небесных тел в пространстве задаются наборами орбитальных элементов: $a, e, i, \omega, \Omega, M$, где $a-$ большая полуось, $e-$ эксцентриситет, $i$ - наклонение, $\omega$ - аргумент перигелия, $\Omega$ - долгота восходящего узла, $M$ - средняя аномалия. Элементы орбит однозначно определяют положение небесного тела. Большая полуось и эксцентриситет задают форму орбиты, наклонение, аргумент перигелия и долготу восходящего узла - ориентацию по отношению к базовой системе координат, средняя аномалия задает положение тела на орбите. В общем случае орбиты тел $A$ и $E$ имеют произвольную ориентацию, то есть параметры $i, \omega, \Omega, M$ отличны от нуля.

Следует отметить, что метод, описанный в данной работе, предназначен для оценки минимального расстояния между орбитами, т. е. данные о реаль- 
ных положениях небесных тел на их орбитах не принимаются во внимание. То есть информация о реальном положении тела на орбите, содержащаяся в орбитальных элементах, в параметре $M$ не используется. Поэтому в дальнейшем вместо шести элементов орбит будет рассматриваться пять. Таким образом, пять элементов орбит будут представлять орбиту небесного тела в пространстве.

Чтобы упростить расчеты, осуществим поворот системы координат таким образом, что орбита тела $E$ не будет наклонена. Элементы орбит тела $A$ необходимо будет пересчитать. Отметим, что такое преобразование не изменяет расстояний между орбитами [12]. Преобразование осуществляется с помощью матрицы перехода $C$, элементы которой зависят от $i_{E}, \omega_{E}, \Omega_{E}$ :

$$
C=\left(\begin{array}{ccc}
\cos \Omega_{E} \cos \omega_{E}-\sin \Omega_{E} \cos i_{E} \sin \omega_{E} & \sin \Omega_{E} \cos \omega_{E}+\cos \Omega_{E} \cos i_{E} \sin \omega_{E} & \sin i_{E} \sin \omega_{E} \\
\cos \Omega_{E} \sin \omega_{E}-\sin \Omega_{E} \cos i_{E} \cos \omega_{E} & \sin \Omega_{E} \sin \omega_{E}+\cos \Omega_{E} \cos i_{E} \cos \omega_{E} & \sin i_{E} \cos \omega_{E} \\
\sin i_{E} \sin \omega_{E} & -\sin i_{E} \cos \Omega_{E} & \cos i_{E}
\end{array}\right) .
$$

Для расчета новых значений $i_{A}, \omega_{A}, \Omega_{A}$ необходимо вычислить следующие величины:

$$
\begin{gathered}
X_{n}=\left(\begin{array}{l}
x_{1 n} \\
x_{2 n} \\
x_{3 n}
\end{array}\right)=C X=\left(\begin{array}{l}
x_{1} c_{11}+x_{2} c_{12}+x_{3} c_{13} \\
x_{1} c_{21}+x_{2} c_{22}+x_{3} c_{23} \\
x_{1} c_{31}+x_{2} c_{32}+x_{3} c_{33}
\end{array}\right) ; \\
Y_{n}=\left(\begin{array}{l}
y_{1 n} \\
y_{2 n} \\
y_{3 n}
\end{array}\right)=C Y=\left(\begin{array}{l}
y_{1} c_{11}+y_{2} c_{12}+y_{3} c_{13} \\
y_{1} c_{21}+y_{2} c_{22}+y_{3} c_{23} \\
y_{1} c_{31}+y_{2} c_{32}+y_{3} c_{33}
\end{array}\right) ; \\
Z_{n}=\left(\begin{array}{l}
z_{1 n} \\
z_{2 n} \\
z_{3 n}
\end{array}\right)=C Z=\left(\begin{array}{l}
z_{1} c_{11}+z_{2} c_{12}+z_{3} c_{13} \\
z_{1} c_{21}+z_{2} c_{22}+z_{3} c_{23} \\
z_{1} c_{31}+z_{2} c_{32}+z_{3} c_{33}
\end{array}\right),
\end{gathered}
$$

где $C$ - матрица перехода, $c_{i j}$ - элементы матрицы $C ; X, Y$ и $Z$ - векторстолбцы, имеющие следующий вид:

$$
X=\left(\begin{array}{c}
\cos \Omega_{A} \cos \omega_{A}-\sin \Omega_{A} \cos i_{A} \sin \omega_{A} \\
\sin \Omega_{A} \cos \omega_{A}+\cos \Omega_{A} \cos i_{A} \sin \omega_{A} \\
\sin i_{A} \sin \omega_{A}
\end{array}\right)
$$

$$
Y=\left(\begin{array}{c}
-\cos \Omega_{A} \sin \omega_{A}-\sin \Omega_{A} \cos i_{A} \cos \omega_{A} \\
-\sin \Omega_{A} \sin \omega_{A}+\cos \Omega_{A} \cos i_{A} \cos \omega_{A} \\
\sin i_{A} \cos \omega_{A}
\end{array}\right), \quad Z=\left(\begin{array}{c}
\sin i_{A} \sin \Omega_{A} \\
-\sin i_{A} \cos \Omega_{A} \\
\cos i_{A}
\end{array}\right) .
$$

Новые значения элементов орбиты тела А рассчитываются по формулам

$$
i_{A}=\arctan \left(\frac{\sqrt{z_{1 n}^{2}+z_{2 n}^{2}}}{z_{3 n}}\right) ; \Omega_{A}=-\arctan \left(\frac{z_{1 n}}{-z_{2 n}}\right) ; \omega_{A}=-\arctan \left(\frac{x_{3 n}}{y_{3 n}}\right) \text {. }
$$

Таким образом, перед началом работы алгоритма получим следующий набор орбитальных элементов: для орбиты тела $E\left(a_{E}, e_{E}, 0,0,0\right)$; для орбиты тела $A\left(a_{A}, e_{A}, i_{A}, \omega_{A}, \Omega_{A}\right)$, где $i_{A}, \omega_{A}, \Omega_{A}$ - пересчитанные по приведенным 
выше формулам элементы орбиты тела $A$ с учетом поворота системы координат [12].

Рассмотрим расположение тел $E$ и $A$ на рис. 1. Солнце-в центре, полярная ось направлена перпендикулярно плоскости орбиты $E$. Плоскость $P$ содержит полярную ось и проходит через тело $A$. Таким образом, плоскость $P$ будет перпендикулярна плоскости орбиты $E$. Так как положение плоскости $P$ зависит от положения тела $A$ на орбите, при движении тела $A$ положение плоскости $P$ будет меняться.

Суть описываемого алгоритма заключается в следующем: производится расчет одного оборота тела $A$ по своей орбите с шагом $\Delta$. В результате получается набор расстояний $D_{v}$, которые можно рассматривать как дискретную функцию. Затем исходя из имеющихся значений функции устанавливаются области локальных минимумов и производится процедура уточнения. При работе первой части алгоритма положение тела $E$ является зависимым от положения тела $A$. То есть положение тела $E$ определяется по положению тела $A$, задаваемому значением истинной аномалии $v_{0}$.

Положение тела $A$ на орбите задается с помощью истинной аномалии $v_{0}$. При этом декартовы координаты тела $A$ и гелиоцентрическое расстояние вычисляются по формулам

$$
\left\{\begin{array}{l}
r_{A 0}=\frac{a_{A}\left(1-e_{A}^{2}\right)}{1+e_{A} \cos v_{0}}, \\
x_{A 0}=r_{A 0}\left(\cos \Omega_{A 0} \cos \left(\omega_{A 0}+v_{0}\right)-\sin \Omega_{A 0} \sin \left(\omega_{A 0}+v_{0}\right) \cos i_{A 0}\right), \\
y_{A 0}=r_{A 0}\left(\sin \Omega_{A 0} \cos \left(\omega_{A 0}+v_{0}\right)-\cos \Omega_{A 0} \sin \left(\omega_{A 0}+v_{0}\right) \cos i_{A 0}\right), \\
z_{A 0}=r_{A 0}\left(\sin \left(\omega_{A 0}+v_{0}\right) \sin i_{A 0}\right) .
\end{array}\right.
$$

Исходя из описанных выше предположений положение тела $E$ на орбите

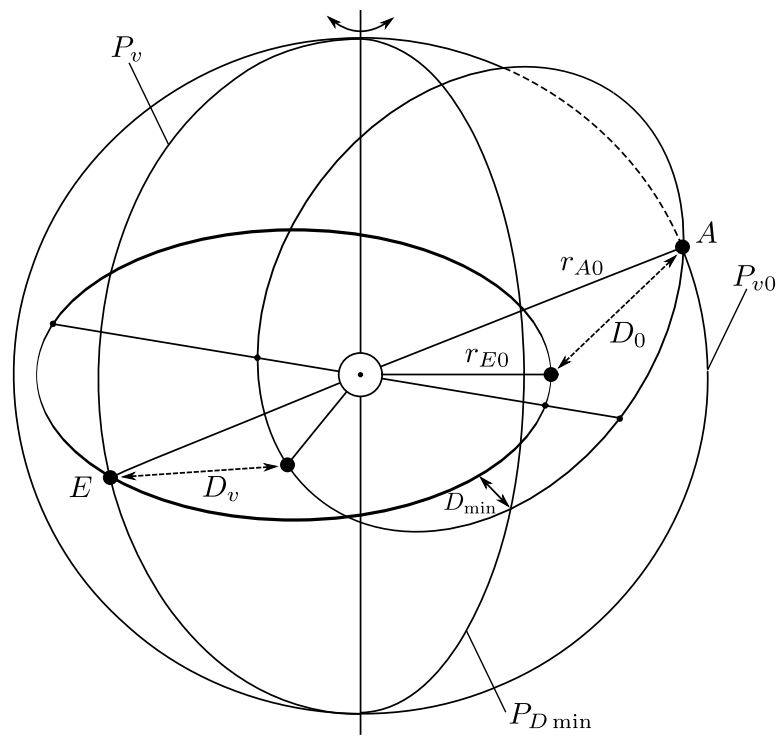

Рис. 1. Расположение орбит тел $E$ и $A$

[Figure 1. Illustration of the orbital geometry of objects $E$ and $A$ ] 
будет определяться положением тела $A$. Плоскость $P$ пересекает орбиту тела $E$ в двух точках. Положим, что тело $E$ находится в той точке, что ближе к телу $A$ (см. рис. 1$)$. Таким образом, Солнце, тело $E$ и тело $A$ образуют треугольник, лежащий в плоскости $P$. Расстояние между $E$ и $A$ обозначим $D_{0}$.

Найдем координаты тела $E$, соответствующие положению тела $A$. Учтем, что в силу вышеупомянутых предположений оба тела находятся вместе с Солнцем в одной плоскости $P_{v 0}$. Обозначим долготу тела $E$ как $L_{0}$, тогда

$$
\cos L_{0}=\frac{x_{A 0}}{\sqrt{x_{A 0}^{2}+y_{A 0}^{2}}}, \quad \sin L_{0}=\frac{y_{A 0}}{\sqrt{x_{A 0}^{2}+y_{A 0}^{2}}}
$$

Так как из-за выбора положения орбиты тела $E$ его долгота совпадает с истинной аномалией, гелиоцентрическое расстояние для тела $E$ вычисляется следующим образом:

$$
r_{A 0}=\frac{a_{E}\left(1-e_{E}^{2}\right)}{1+e_{E} \cos L_{0}} .
$$

Теперь, используя геометрические соображения относительно положений тел, найдем расстояние между телами $A$ и $E$ в их текущих положениях. На рис. 1 этим положениям соответствует расстояние $D_{0}$. Рассмотрим треугольник, образованный телами и Солнцем в плоскости $P_{v 0}$ (см. рис. 2).

В треугольнике $S A E$ две стороны равны гелиоцентрическим расстояниям $r_{A 0}$ и $r_{E 0}$, а третья - искомому расстоянию $D_{0}$. Опустим перпендикуляр из точки $A$ на продолжение луча $S E$, получим точку $A^{\prime}$. Учитывая, что точка $A$ имеет координаты $\left(x_{A 0}, y_{A 0}, z_{A 0}\right)$, рассчитанные по формулам $(1)$, получим значение $S A^{\prime}$ :

$$
S A^{\prime}=\sqrt{x_{A 0}^{2}+y_{A 0}^{2}}
$$

Рассмотрим треугольник $E A^{\prime} A$, в котором $E A^{\prime}=S A^{\prime}-S E$. Очевидно,

$$
E A^{\prime}=\sqrt{\left(x_{A 0}^{2}+y_{A 0}^{2}\right)^{2}}-r_{E 0}
$$

Сторона $A A^{\prime}$ равна $z_{A 0}$. В свою очередь, сторона $E A$ является гипотенузой прямоугольного треугольника, а значит

$$
E A^{2}=E A^{\prime 2}+A A^{2}
$$

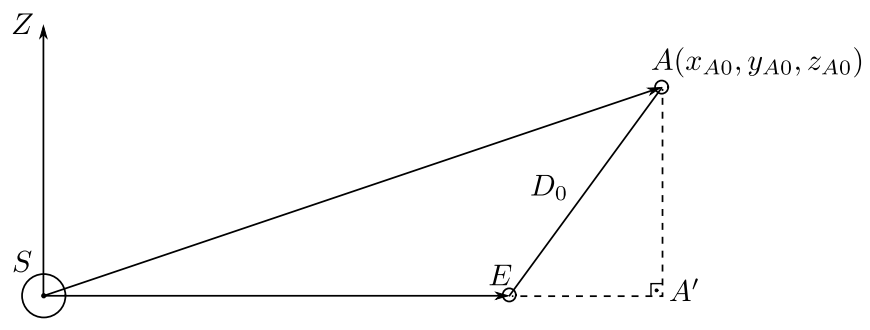

Рис. 2. Расположение тел $E$ и $A$ при определении расстояния между орбитами [Figure 2. The triangle $S A E$ for calculating the distance between orbits of $E$ and $A$ ] 
Получается, что искомое значение $D_{0}$, соответствующее длине стороны $E A$ треугольника $E A^{\prime} A$, может быть вычислено следующим образом:

$$
D_{0}^{2}=z_{A 0}^{2}+\left(\sqrt{x_{A 0}^{2}+y_{A 0}^{2}}-r_{E 0}\right)^{2} .
$$

После вычисления $D_{0}$ расчет для одного положения тела $A$ на орбите окончен. Теперь необходимо изменить положение тела $A$ на орбите, увеличив истинную аномалию $v_{0}$ на величину $\Delta$, и повторить вычисления. Таким образом, получим очередное значение расстояния, например, $D_{v}$ (см. рис. 1 ).

Проводя описанные выше вычисления до полного оборота тела $A$ по орбите, получим набор расстояний между орбитами тел $A$ и $E$ как дискретную функцию, зависящую от $v$, для которой можно установить точки локальных минимумов. Один из примеров локального минимума $D_{\min }$ изображен на рис. 1.

Таким образом, первая стадия алгоритма заключается в поиске областей, содержащих локальные минимумы расстояний между орбитами тел $E$ и $A$.

Вторая стадия алгоритма состоит в уточнении локальных минимумов расстояний между орбитами и поиске MOID. Исходя из данных о точках локального минимума и соответствующих им координат тел $E$ и $A$, зафиксированных на первой стадии работы алгоритма, производится уточнение данных и поиск среди уточненных данных глобального минимума, который и будет являться оценкой значения MOID.

Допустим, в результате первой фазы работы алгоритма найдено $N$ предварительных локальных минимумов. Имеем $N$ положений тел $E$ и $A$ и $2 N$ наборов орбитальных элементов. Требуется произвести уточнение позиций $E$ и $A$ для каждого найденного локального минимума $D_{v}$.

Пусть имеем положения $E_{1}$ и $A_{1}$, а также соответствующее им расстояние между орбитами $D_{\min }$. Добавим к рассмотрению еще 4 точки: $E_{1+}, E_{1-}$, $A_{1+}, A_{1-}$. Эти положения получаются путем варьирования истинной аномалии тела на величину $\Delta v$, т.е. сдвига по направлению движения (индекс + ) и против движения (индекс -) на фиксированную величину $\Delta v$. Таким образом, получим 6 позиций и 9 расстояний между орбитами $E$ и $A$.

Расстояния между телами $E$ и $A$ вычисляются как расстояния между двумя точками в декартовой трехмерной системе координат с использованием тривиальных формул:

$$
\begin{aligned}
& \left\{\begin{array}{l}
r_{A}=\frac{a_{E}\left(1-e_{E}^{2}\right)}{1+e_{E} \cos L_{E}} \\
x_{E}=r_{E} \cos L_{E} \\
y_{E}=r_{E} \sin L_{E} \\
z_{E}=0
\end{array}\right. \\
& \left\{\begin{array}{l}
r_{A}=\frac{a_{A}\left(1-e_{A}^{2}\right)}{1+e_{A} \cos v_{A}} \\
x_{A}=r_{A}\left(\cos \Omega_{A} \cos \left(\omega_{A}+v_{A}\right)-\sin \Omega_{A} \sin \left(\omega_{A}+v_{A}\right) \cos i_{A}\right) ; \\
y_{A}=r_{A}\left(\sin \Omega_{A} \cos \left(\omega_{A}+v_{A}\right)-\cos \Omega_{A} \sin \left(\omega_{A}+v_{A}\right) \cos i_{A}\right) ; \\
z_{A}=r_{A}\left(\sin \left(\omega_{A}+v_{A}\right) \sin i_{A}\right),
\end{array}\right.
\end{aligned}
$$




$$
D^{2}=\left(x_{A}-x_{E}\right)^{2}+\left(y_{A}-y_{E}\right)^{2}+\left(z_{A}-z_{E}\right)^{2},
$$

где $L_{E}$ и $v_{A}$ - истинные аномалии тел $E$ и $A$ соответственно (с учетом изменений их на величину $\Delta v)$.

Важно заметить, что на второй итерации цикла количество расстояний, которые необходимо будет вычислить, сократится. $\mathrm{K}$ примеру, если новому положению тел $E$ и $A$ будут соответствовать точки $E_{1-}, A_{1-}$, то добавятся только 2 новых положения тел (т.к. остальные были вычислены на предыдущем шаге).

Вторая фаза алгоритма повторяется с учетом того, что положения $E_{1}$ и $A_{1}$ равны положениям, соответствующим новому минимуму расстоянию $D_{\min } 1$. Цикл продолжается, пока на $k$-том шаге цикла найденное минимальное расстояние не совпадет с заданной точностью с найденным на шаге $k-1$.

Описанный выше алгоритм уточнения выполняется для каждого из $N$ локальных минимумов, найденных на первой стадии работы алгоритма. Вторая стадия повторяется несколько раз с уменьшением шага $\Delta v$. После завершения второй стадии алгоритма минимальное расстояние между орбитами

$$
\text { MOID }=\min _{i=1, \ldots N} D_{\min i}
$$

Были проведены сравнительные испытания алгоритма с классическим методом G. F. Gronchi, исходный код которого находится в общем доступе (http://adams.dm.unipi.it/?gronchi/kepdist). Начальные данные астероидов брались с сайта Центра малых планет Международного астрономического союза (http://www.minorplanetcenter.net/iau/Dangerous.html), a также с научно-информационного сайта SmallBodies.ru (http: //smallbodies . ru). Результаты испытаний приведены в табл. 1 и табл. 2.

В табл. 1 приведены результаты оценки количества потенциально опасных астероидов (PHA - Potentially Hazardous Asteroids) среди групп Аполлона, Амура и Атона с помощью метода, описанного в настоящей статье. Астероиды этих групп выбраны в силу того, что почти все потенциально опасные астероиды, известные на сегодняшний день, относятся к одной из этих трех групп. Результаты работы метода совпали с данными, публикуемыми Лабораторией реактивного движения NASA (http://neo.jpl.nasa.gov/orbits) и Центром малых планет международного астрономического союза. Как видно из полученных данных, наибольшее число потенциально опасных астероидов находится в группе Аполлона. Отметим, что при составлении табл. 1 переход

Таблица 1

Потенциально опасные астероиды в группах Аполлона, Амура и Атона [Potentially hazardous asteroids found in Apollo, Amor, and Aten groups]

\begin{tabular}{c|c|c|c}
\hline $\begin{array}{c}\text { Группа астероидов } \\
\text { [Group of asteroids] }\end{array}$ & $\begin{array}{c}\text { Количество астероидов } \\
\text { [Number of asteroids] }\end{array}$ & $\begin{array}{c}\text { Количество PHA } \\
\text { [Number of PHA] }\end{array}$ & $\begin{array}{c}\text { Процент PHA } \\
\text { [Percent of PHA] }\end{array}$ \\
\hline Аполлоны [Apollo] & 6580 & 1319 & $20.05 \%$ \\
Амуры [Amor] & 4726 & 83 & $1.76 \%$ \\
Атоны [Aten] & 908 & 145 & $15.97 \%$ \\
По трем группам & 12214 & 1547 & $12.67 \%$ \\
[Into three groups] & & & \\
\hline
\end{tabular}




\section{Таблица 2}

Сравнение точности расчетов MOID [Comparison of the MOID calculation accuracy]

\begin{tabular}{c|c|c}
\hline Астероид [Asteroid] & MOID, a.u. & $\Delta, 10^{-13}$ a.u. \\
\hline 2000 SG344 & 0.000790777 & 1.5 \\
410777 (2009 FD) & 0.002290780 & 5.9 \\
99942 Apophis & 0.000659446 & 10.4 \\
6344 P-L & 0.028124800 & 0.7 \\
2005 WG57 & 0.001624190 & 4.8 \\
\hline
\end{tabular}

астероидов из одной группы в другую во время эволюции орбиты (к примеру, в результате тесных сближений) не рассматривался.

Следует отметить, что при сравнении скорости работы в методе, описанном в данной работе, не использовались механизмы параллельных вычислений, которые могли бы еще больше ускорить процедуру расчета MOID. Скорость расчетов составляла в среднем 12 ms на 100 вычислений MOID для описанного алгоритма против $40 \mathrm{~ms}$ в среднем на 100 вычислений MOID по методу G. F. Gronchi.

В табл. 2 приводятся значения параметра MOID, рассчитанные для нескольких потенциально опасных астероидов. В силу того, что при расчете параметра MOID по методу, описанному в данной статье, получаются значения, совпадающие с классическим методом G. F. Gronchi как минимум до 9 разряда, в табл. 2 во втором столбце приведено значение MOID с точностью до 9-го знака, а в третьем - разность $\Delta=\mid \mathrm{MOID}_{\mathrm{Gr}}-\mathrm{MOID}$ Fast $\mid$, где MOID $_{\mathrm{Gr}}$ - значение, полученное по методу G. F. Gronchi, a MOID Fast - значение, полученное по описанному в статье алгоритму. Таким образом, видно, что в результате настройки количества итераций в первой и второй частях алгоритма (параметры $\Delta$ и $\Delta v$ ) можно добиться совпадения значений MOID с рассчитанными по методу G. F. Gronchi до значений порядка от $10^{-12}$ до $10^{-14}$.

Характеристики рабочей станции, на которой проводились сравнительные испытания методов: CPU: Intel Core i7-4800MQ (4 kernels, 2.7 GGz); RAM: 8.0 GiB, DDR3; HDD: 1 TiB, 7200 rpm.; OS: Windows 8.164 bit.

Метод, описанный в данной работе, может применяться не только для определения минимального расстояния между орбитами Земли и астероида, но и для расчета минимального расстояния между орбитами двух произвольных небесных тел. Как уже было отмечено выше, единственным условием остается требование конфокальности эллиптических орбит двух небесных тел.

Представленный метод расчета MOID, в отличие от аналитических методов K. В. Холшевникова и G. F. Gronchi, не предоставляет информацию об особых точках функции, описывающей расстояние между орбитами $E$ и $A$ (максимумы расстояний, седловые точки). Однако так как метод создан для оценки только параметра MOID для большого количества астероидов с высокой скоростью и точностью, данная информация для поставленных задач не требуется.

\section{ORCID}

Андрей Евгеньевич Деревянка: http://orcid.org/0000-0003-1682-7873 


\section{БИБЛИОГРАФИЧЕСКИЙ СПИСОК}

1. Tancredi G. A criterion to classify asteroids and comets based on the orbital parameters // Icarus, 2014. vol.234. pp. 66-80. doi: 10.1016/j.icarus.2014.02.013.

2. Milani A., Chesley S. R., Valsecchi G. B. Asteroid close encounters with Earth: Risk assessment // Planetary and Space Science, 2000. vol. 48, no. 10. pp. 945-954. doi: 10.1016/ s0032-0633(00)00061-1.

3. Milani A. The asteroid identification problem I. Recovery of lost asteroids // Icarus, 1999. vol. 137, no. 2. pp. 269-292. doi: 10.1006/icar.1999.6045.

4. Sitarski G. Approaches of the parabolic comets to the outer planets // Acta Astronomica, 1968. vol. 18, no. 2. pp. 171-195.

5. Milani A., Chesley S. R., Valsecchi G. B. Asteroid Close Approaches: Analysis and Potential Impact Detection / Asteroids III; eds. W. Bottke, A. Cellino, P. Paolicchi, and R. P. Binzel: University of Arizona Press, 2002. pp. 55-69.

6. Kholshevnikov K. V., Vassiliev N. N. On the distance function between two Keplerian elliptic orbits // Celestial Mechanics and Dynamical Astronomy, 1999. vol. 75, no. 2. pp. 7583. doi : 10.1023/A:1008312521428.

7. Baluyev R. V., Kholshevnikov K. V. Distance between two arbitrary unperturbed orbits // Celestial Mechanics and Dynamical Astronomy, 2005. vol. 91, no. 3-4. pp. 287-300. doi : 10. 1007/s10569-004-3207-1.

8. Gronchi G. F., Tommei G., Milani A. Mutual geometry of confocal Keplerian orbits: uncertainty of the MOID and search for virtual PHAs // Proceedings of the International Astronomical Union, 2006. vol. 2, no. S236. pp. 3-14. doi : 10.1017/s1743921307003018.

9. Gronchi G. F. An Algebraic Method to Compute the Critical Points of the Distance Function Between Two Keplerian Orbits// Celestial Mechanics and Dynamical Astronomy, 2005. vol. 93, no. 1-4. pp. 295-329. doi: 10.1007/s10569-005-1623-5.

10. Gronchi G. F. On the stationary points of the squared distance between two ellipses with a common focus // SIAM J. Sci. Comput., 2002. vol.20, no.1. pp. 61-80. doi: 10.1137/ s1064827500374170.

11. Armellin R., Di Lizia P., Berz M., Makino K. Computing the critical points of the distance function between two Keplerian orbits via rigorous global optimization // Celestial Mechanics and Dynamical Astronomy, 2010. vol.107, no.3. pp. 377-395. doi: 10.1007/ s10569-010-9281-7.

12. Wiśniowski T., Rickman H. Fast Geometric Method for Calculating Accurate Minimum Orbit Intersection Distances (MOIDs) // Acta Astronomica, 2013. vol. 63, no. 2. pp. 293307.

13. Vasile M., Colombo C. Optimal Impact Strategies for Asteroid Deflection // Journal of Guidance, Control and Dynamics, 2008. vol.31, no.4. pp. 858-872. doi: 10.2514/1.33432.

14. Besse I. M., Rhee N. H. A numerical method for calculating minimum distance to Near Earth Objects // Applied Mathematics and Computation, 2014. vol.237. pp. 274-281. doi: 10. 1016/j.amc.2014.03.115.

15. Marčeta D., Šegan S. The distributions of positions of Minimal Orbit Intersection Distances among Near Earth Asteroids // Advances in Space Research, 2012. vol. 50, no. 2. pp. 256-259. doi: 10.1016/j.asr.2012.04.005.

16. Carusi A., Dotto E. Close Encounters of Minor Bodies with the Earth // Icarus, 1996. vol. 124, no. 2. pp. 392-398. doi: 10.1006/icar.1996.0216.

17. Milisavljevic S. The proximities of asteroids and critical points of the distance function // Serbian Astronomical Journal, 2010. vol. 180. pp. 91-102. doi: 10.2298/saj1080091m.

18. Šegan S., Milisavljević S., Marčeta D. A combined method to compute the proximities of asteroids // Acta Astronomica, 2011. vol. 61, no. 3. pp. 275-283.

19. Hoots F. R., Crawford L. L., Roehrich R. L. An analytical method to determine future close approaches between satellites // Celestial Mechanics and Dynamical Astronomy, 1984. vol. 33, no. 2. pp. 143-158. doi: 10.1007/bf01234152. 
20. Dybczyński P. A., Jopek T. J., Serafin R. A. On the minimum distance between two Keplerian orbits with a common focus // Celestial Mechanics and Dynamical Astronomy, 1986. vol. 38, no. 4. pp. 345-356. doi: 10.1007/bf01238925.

Поступила в редакцию $15 / \mathrm{X} / 2014$;

в окончательном варианте - $12 / \mathrm{XI} / 2014$;

принята в печать $-27 / \mathrm{XI} / 2014$.

Vestn. Samar. Gos. Techn. Un-ta. Ser. Fiz.-mat. nauki

[J. Samara State Tech. Univ., Ser. Phys. \& Math. Sci.] 2014. Issue 4 (37). Pp. 144-156

ISSN: 2310-7081 (online), 1991-8615 (print)

doi: http://dx.doi.org/10.14498/vsgtu1344

MSC: 70-08, 70M20; 70F05, 70F15

\title{
A METHOD FOR THE FAST MOID COMPUTATION FOR TWO CONFOCAL HELIOCENTRIC ORBITS
}

\section{A. E. Derevyanka}

Samara State Technical University,

244, Molodogvardeyskaya st., Samara, 443100, Russian Federation.

\begin{abstract}
The paper is on the problem of classification an asteroid as potentially hazardous (PHA), namely the estimation of the MOID parameter. Minimum Orbital Intersection Distance describes the minimal distance between two confocal heliocentric orbits. Analytical, numerical and hybrid methods used for the MOID estimation are reviewed. A brief description of the K. V. Kholshevnikov and G. F. Gronchi analytical methods, which are considered to be classical, is given. The task of calculating the MOID parameter for a large number of asteroids (more than 10,000) with a maximum calculating speed and the ability to parallelize the process is set. A numerical method based on geometrical considerations concerning the location of the bodies on their orbits is proposed. Let us consider two bodies $A$ and $E$. Since only the minimum distance between two orbits is required, the information on the actual position of the bodies on their orbits is insignificant. The idea is to calculate one full revolution of the body $A$. For each position of body $A$ the corresponding position of the body $E$ is calculated under the following assumption. Consider a plane $P$, comprising the body $A$ and the Sun. Therefore, plane $P$ is perpendicular to the orbital plane of the body $E$. Of the two points at which the plane $P$ intersects the orbit of the body $E, E$ is considered to be at the point that is the nearest the body $A$. Thus, the position of the body $E$ will depend on the position of the body $A$. As a result, from the geometric assumptions on the triangle formed by the Sun and two bodies, the distance between $A$ and $E$ is calculated. When one complete revolution of the body $A$ with a certain step is calculated, we receive a set
\end{abstract}

(C) 2014 Samara State Technical University.

How to cite Reference

Derevyanka A. E. A method for the fast MOID computation for two confocal heliocentric orbits, Vestn. Samar. Gos. Tekhn. Univ., Ser. Fiz.-Mat. Nauki [J. Samara State Tech. Univ., Ser. Phys. \& Math. Sci.], 2014, no. 4 (37), pp. 144-156. doi: 10.14498/vsgtu1344. (In Russian) Author Details

Andrey E. Derevyanka (AndrDerev@gmail.com), Postgraduate Student, Dept. of Applied Mathematics \& Computer Science. 
of the distances between two orbits, from which we can identify the areas of the local minima of the discrete representation of the distance function (the distance between the orbits of $A$ and $E$ ). Then, the procedure of tuning is carried out to verify and precise the values of local minima of discrete representation of the distance function. As a result, the smallest value of the local minima is considered to be the estimation of the Minimum Orbital Intersection Distance (MOID) takes. Pros of the suggested method are as follows: high speed and adjustable calculation accuracy, the suitability to the use of parallel computing. Comparative tests of the described method were carried out. The results received are consistent with the classical G. F. Gronchi method.

Keywords: MOID, potentially hazardous asteroids, orbital elements, celestial mechanics.

doi: http://dx.doi.org/10.14498/vsgtu1344

\section{ORCID}

Andrey E. Derevyanka: http://orcid.org/0000-0003-1682-7873

\section{REFERENCES}

1. Tancredi G. A criterion to classify asteroids and comets based on the orbital parameters, Icarus, 2014, vol. 234, pp. 66-80. doi: 10.1016/j.icarus.2014.02.013.

2. Milani A., Chesley S. R., Valsecchi G. B. Asteroid close encounters with Earth: Risk assessment, Planetary and Space Science, 2000, vol.48, no. 10, pp. 945-954. doi: 10.1016/ s0032-0633(00)00061-1.

3. Milani A. The asteroid identification problem I. Recovery of lost asteroids, Icarus, 1999, vol. 137, no. 2, pp. 269-292. doi: 10.1006/icar.1999.6045.

4. Sitarski G. Approaches of the parabolic comets to the outer planets, Acta Astronomica, 1968, vol. 18, no. 2, pp. 171-195.

5. Milani A., Chesley S. R., Valsecchi G. B. Asteroid Close Approaches: Analysis and Potential Impact Detection, Asteroids III; eds. W. Bottke, A. Cellino, P. Paolicchi, and R. P. Binzel, University of Arizona Press, 2002, pp. 55-69.

6. Kholshevnikov K. V., Vassiliev N. N. On the distance function between two Keplerian elliptic orbits, Celestial Mechanics and Dynamical Astronomy, 1999, vol.75, no. 2, pp. 7583. doi : 10.1023/A: 1008312521428.

7. Baluyev R. V., Kholshevnikov K. V. Distance between two arbitrary unperturbed orbits, Celestial Mechanics and Dynamical Astronomy, 2005, vol. 91, no. 3-4, pp. 287-300. doi : 10. 1007/s10569-004-3207-1.

8. Gronchi G. F., Tommei G., Milani A. Mutual geometry of confocal Keplerian orbits: uncertainty of the MOID and search for virtual PHAs, Proceedings of the International Astronomical Union, 2006, vol. 2, no. S236, pp. 3-14. doi : 10.1017/s1743921307003018.

9. Gronchi G. F. An Algebraic Method to Compute the Critical Points of the Distance Function Between Two Keplerian Orbits, Celestial Mechanics and Dynamical Astronomy, 2005, vol. 93, no. 1-4, pp. 295-329. doi: 10.1007/s10569-005-1623-5.

10. Gronchi G. F. On the stationary points of the squared distance between two ellipses with a common focus, SIAM J. Sci. Comput., 2002, vol.20, no.1, pp. 61-80. doi: 10.1137/ s1064827500374170.

11. Armellin R., Di Lizia P., Berz M., Makino K. Computing the critical points of the distance function between two Keplerian orbits via rigorous global optimization, Celestial Mechanics and Dynamical Astronomy, 2010, vol.107, no.3, pp. 377-395. doi:10.1007/ s10569-010-9281-7.

12. Wiśniowski T., Rickman H. Fast Geometric Method for Calculating Accurate Minimum Orbit Intersection Distances (MOIDs), Acta Astronomica, 2013, vol. 63, no. 2, pp. 293-307. 
13. Vasile M., Colombo C. Optimal Impact Strategies for Asteroid Deflection, Journal of Guidance, Control and Dynamics, 2008, vol.31, no. 4, pp. 858-872. doi: 10.2514/1.33432.

14. Besse I. M., Rhee N. H. A numerical method for calculating minimum distance to Near Earth Objects, Applied Mathematics and Computation, 2014, vol. 237, pp. 274-281. doi : 10.1016/ j.amc.2014.03.115.

15. Marčeta D., Šegan S. The distributions of positions of Minimal Orbit Intersection Distances among Near Earth Asteroids, Advances in Space Research, 2012, vol. 50, no. 2, pp. 256-259. doi: 10.1016/j.asr.2012.04.005.

16. Carusi A., Dotto E. Close Encounters of Minor Bodies with the Earth, Icarus, 1996, vol. 124, no. 2, pp. 392-398. doi: 10.1006/icar.1996.0216.

17. Milisavljevic S. The proximities of asteroids and critical points of the distance function, Serbian Astronomical Journal, 2010, vol. 180, pp. 91-102. doi: 10.2298/saj1080091m.

18. Šegan S., Milisavljević S., Marčeta D. A combined method to compute the proximities of asteroids, Acta Astronomica, 2011, vol. 61, no. 3, pp. 275-283.

19. Hoots F. R., Crawford L. L., Roehrich R. L. An analytical method to determine future close approaches between satellites, Celestial Mechanics and Dynamical Astronomy, 1984, vol. 33, no. 2, pp. 143-158. doi: 10.1007/bf01234152.

20. Dybczyński P. A., Jopek T. J., Serafin R. A. On the minimum distance between two Keplerian orbits with a common focus, Celestial Mechanics and Dynamical Astronomy, 1986, vol. 38, no. 4, pp. 345-356. doi: 10.1007/bf01238925.

Received 15/X/2014;

received in revised form $12 / \mathrm{XI} / 2014$;

accepted 27/XI/2014. 